\title{
Lung transplantation and concomitant cardiac surgery: Is it justified?
}

\author{
Reshma Biniwale, MD, ${ }^{\mathrm{a}}$ David Ross, MD, ${ }^{\mathrm{b}}$ Amit Iyengar, MS, ${ }^{\mathrm{a}}$ Oh Jin Kwon, BS, ${ }^{\mathrm{a}}$ \\ Curtis Hunter, MD, ${ }^{\mathrm{a}}$ Jamil Aboulhosn, MD, ${ }^{\mathrm{c}}$ David Gjertson, $\mathrm{PhD},{ }^{\mathrm{d}}$ and Abbas Ardehali, MD ${ }^{\mathrm{a}}$
}

\begin{abstract}
Objective: Increasing numbers of lung transplant candidates have cardiac conditions that affect their survival after transplantation. Our objective was to determine if patients who undergo concomitant cardiac surgery (CCS) during the lung transplant procedure have similar outcomes, as a cohort of isolated lung transplant recipients.
\end{abstract}

Methods: This was a retrospective, observational, matched-cohort analysis. The records of lung transplant recipients who underwent CCS from August 2000 to August 2013 were reviewed. A cohort of isolated lung transplant recipients, matched on the basis of age, lung allocation score, diagnosis, type of procedure, and era, was identified. The primary endpoint of this trial was 5-year survival. The secondary endpoints were primary graft dysfunction, grade III, at 72 hours, intensive care unit and hospital length of stay, and 5-year major adverse cardiac event rates.

Results: A total of 120 patients underwent lung transplantation and CCS. Compared with the isolated lung transplant group, the donor, recipient, and operation characteristics were similar. No difference was found in the survival of the 2 groups for up to 5 years, or in the incidence of primary graft dysfunction Grade III at 72 hours, intensive care unit length of stay, invasive ventilation, hospital length of stay, or incidence of 5-year major adverse cardiac events.

Conclusions: Lung transplant recipients undergoing CCS have early and midterm clinical outcomes similar to those of isolated lung transplant recipients. Given that this report is the largest published experience, offering cardiac surgery at the time of lung transplantation, to selected patients, remains justified. (J Thorac Cardiovasc Surg 2016;151:560-7)
Lung transplantation is now an accepted form of therapy in many patients who have end-stage lung diseases. With improved peritransplant care and posttransplant outcomes, many patients previously deemed ineligible are now considered for lung transplantation. Many of these recipients have

From the Divisions of ${ }^{\mathrm{a} C}$ Cardiac Surgery, and ${ }^{\mathrm{b}} \mathrm{Pulmonology}$, University of California, Los Angeles Medical Center; ${ }^{\mathrm{c}}$ Division of Adult Congenital Cardiology, and ${ }^{\mathrm{d}}$ Pathology \& Laboratory Medicine and Biostatistics, University of California, Los Angeles, Los Angeles, Calif.

Read at the 95th Annual Meeting of The American Association for Thoracic Surgery, Seattle, Washington, April 25-29, 2015.

Received for publication April 16, 2015; revisions received Aug 5, 2015; accepted for publication Oct 1, 2015; available ahead of print Nov 24, 2015.

Address for reprints: Reshma Biniwale, MD, Division of Cardiac Surgery, UCLA rbiniwale@mednet.ucla.edu).

$0022-5223 / \$ 36.00$

Copyright (c) 2016 by The American Association for Thoracic Surgery http://dx.doi.org/10.1016/j.jtcvs.2015.10.027 Medical Center, CHS 62-266, Le Conte Ave, Los Angeles, CA 90095 (E-mail:

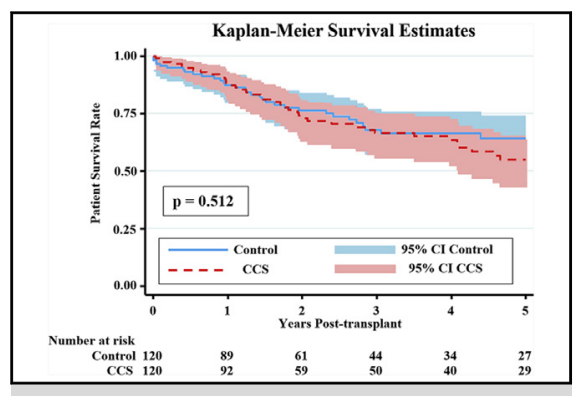

Survival of lung transplant recipients who had, versus did not have, concomitant cardiac surgery.

\section{Central Message}

Lung transplantation and concomitant cardiac surgery can be performed with clinical outcomes similar to those for isolated lung transplantation.

\section{Perspective}

Increasingly, lung transplant candidates have concomitant cardiac conditions that require cardiac surgical repair. Our study suggests that lung transplant recipients who require CCS have early and midterm outcomes similar to those of isolated lung transplant recipients. The implications of this study need to be weighed against the scarcity of donor organs.

See Editorial Commentary page 568.

See Editorial page 315. comorbidities that may affect their posttransplant outcomes. ${ }^{1}$ Given the limited number of donors, acceptance of potential recipients with comorbidities (with unknown or possibly inferior clinical outcomes) becomes an ethical dilemma.

An increasing number of patients being considered for lung transplantation have cardiac conditions that require repair prior to, or concomitant with, lung transplantation. ${ }^{1,2}$ Most of these patients are not candidates for cardiac surgery prior to transplantation, owing to the severity of their lung disease. Concomitant cardiac surgery (CCS) and lung transplantation often necessitate cardiopulmonary bypass (in most cases), prolong operative time, and can affect short- and medium-term posttransplant outcomes.

Several reports have examined the clinical outcomes of lung transplantation and concomitant coronary revascularization procedures. ${ }^{3-5}$ Information is limited on the clinical outcomes of lung transplantation in patients who need 


\section{Abbreviations and Acronyms \\ $\mathrm{CABG}=$ coronary artery bypass grafting \\ CCS = concomitant cardiac surgery}

CCS. A contemporary report with a large number of patients will broaden the knowledge base and rationalize the current policy of offering lung transplantation to patients who have concomitant cardiac diseases.

Our program has offered lung transplantation and CCS for $>10$ years. We hypothesized that in highly selected patients who need corrective cardiac procedures, simultaneous lung transplantation and CCS can be performed with clinical outcomes comparable to those for patients undergoing isolated lung transplantation. The purpose of this report is to compare 5-year survival; postoperative clinical outcomes; and 5-year major adverse cardiac events of patients who underwent lung transplantation and CCS (CCS group), compared with a matched group who underwent lung transplantation alone (control group).

\section{METHODS}

Institutional review board approval was given for this study. The records of 620 patients were reviewed who had undergone lung transplantation in the period from August 2000 to August 2013. Of these, 120 had undergone CCS; this cohort was the subject of this report.

\section{Cardiac Evaluation in Lung Transplantation Candidates}

All potential lung transplantation candidates were screened with a transthoracic echocardiogram. Male patients aged $>40$ years, female patients aged $>45$ years, and younger patients who had symptoms suggestive of coronary artery disease underwent coronary angiography to rule out coronary artery disease.

\section{Selection of Patients for Lung Transplantation and Concomitant Cardiac Surgery}

Patients with patent foramen ovale or atrial septal defects; $\geq$ moderate tricuspid regurgitation; tricuspid annulus $>4 \mathrm{~cm}$ or $>2.1 \mathrm{~cm} / \mathrm{m}^{2}$ (irrespective of pulmonary artery pressure); $\geq$ moderate mitral regurgitation; $\geq$ moderate aortic stenosis; and normal ejection fraction, all on transthoracic echocardiogram (valve area $<1.5 \mathrm{~m}^{2}$ ), were considered for lung transplantation and CCS. Patients with discrete coronary artery disease and preserved left ventricle ejection fraction were additionally considered for lung transplantation and concomitant coronary artery bypass grafting (CABG; part of this group's clinical outcome has been previously reported). ${ }^{3}$ The presence of a cardiac condition necessitating concomitant repair was considered within the context of each patient's candidacy. Patients with several relative contraindications (ie, age $>70$ years) and in need of CCS were declined. Major CCS was defined as cardiac surgical procedures excluding isolated patent foramen ovale repair.

\section{Inclusion and Exclusion Criteria}

All patients who underwent lung transplantation and CCS during the study period were included in the study. This group included redo lung transplantation recipients. Pediatric (age $<18$ years), and multivisceral transplantation, patients were excluded.

\section{Control Group}

Each lung transplant recipient who underwent CCS was matched to an isolated lung transplant recipient (1:1 ratio). Propensity-score matching was performed based on recipient age, era (within 1 year of the procedure), pulmonary diagnosis, lung allocation score, and the type of lung transplantation procedure.

\section{Peri- and Post-Operative Care}

The timing of surgery for patients in need of lung transplantation and CCS was coordinated such that the cardiac procedure was usually done first (prior to arrival of the organ). Our strategy for coronary revascularization has been previously reported. ${ }^{3}$ Patients who require tricuspid valve intervention underwent annuloplasty with either a pericardial strip or an Edwards MC3 tricuspid ring (Model 4900; Edwards Lifesciences, Irvine, Calif) annuloplasty. Patients with an annuloplasty ring were maintained on warfarin for 3 months.

The immunosuppressive regimen for all patients included induction therapy, with either: Thymoglobulin (Genzyme, Cambridge, Mass), for patients aged $<60$ years in absence of infectious lung diseases; or basiliximab, for the remaining recipients. All lung transplant recipients received tacrolimus, mycophenolate mofetil, and steroids. All patients were treated with pravastatin, and aspirin (unless being treated with warfarin for the first 3 months) indefinitely.

\section{Data Endpoints}

The following donor characteristics were collected: age, gender, body mass index, diabetes, cytomegalovirus status, smoking history, last ratio of arterial oxygen partial pressure to fractional inspired oxygen (prior to harvest), and cause of death. The collected recipient characteristics were as follows: age, gender, height, weight, body mass index, diabetes, systemic hypertension, cytomegalovirus status, 6-minute walk test, etiology of lung failure (obstructive, cystic fibrosis/bronchiectasis, pulmonary fibrosis, or pulmonary vascular disease), systolic, diastolic, and mean pulmonary artery pressures, right atrial pressure, pulmonary vascular resistance, cardiac output/index, lung allocation score, and days on the waitlist. In addition, the following data points were collected in reference to the transplantation procedure: type of procedure (single vs double lung transplant); donor/recipient gender mismatch; donor/recipient cytomegalovirus mismatch; allograft ischemia time; number performed on bypass; cardiopulmonary bypass time; and aortic crossclamp time.

The primary endpoint for this study was 5-year patient survival. Survival information was confirmed at the end of the study period. This study had 2 secondary endpoints: (1) postoperative clinical outcomes; and (2) 5-year major adverse cardiac events. The postoperative clinical outcomes that were collected and analyzed were as follows: duration of invasive mechanical ventilation, incidence of re-exploration for bleeding, incidence of operative wound revision, requirement for postoperative extracorporeal membrane oxygenation support, primary graft dysfunction grade III at 72 hours, intensive care unit length of stay, hospital length of stay, and 30-day or in-hospital mortality. ${ }^{6}$

In-hospital cardiac complications, including the incidence of atrial fibrillation (lasting for $>30$ minutes and requiring treatment during the same hospital admission or within 30 days of surgery), myocardia infarction, and cerebrovascular accident were collected and analyzed. The category of 5-year major adverse cardiac events was defined as: episodes of atrial fibrillation (documented episode requiring treatment); episodes of acute coronary syndrome; redo revascularization or valvular procedure; episodes of congestive heart failure (requiring hospital admission); and cardiac-related deaths, including pulmonary embolism.

\section{Statistical Analysis}

Our matching procedure was performed using propensity-score analysis. Variables selected for the matching procedure were chosen based 


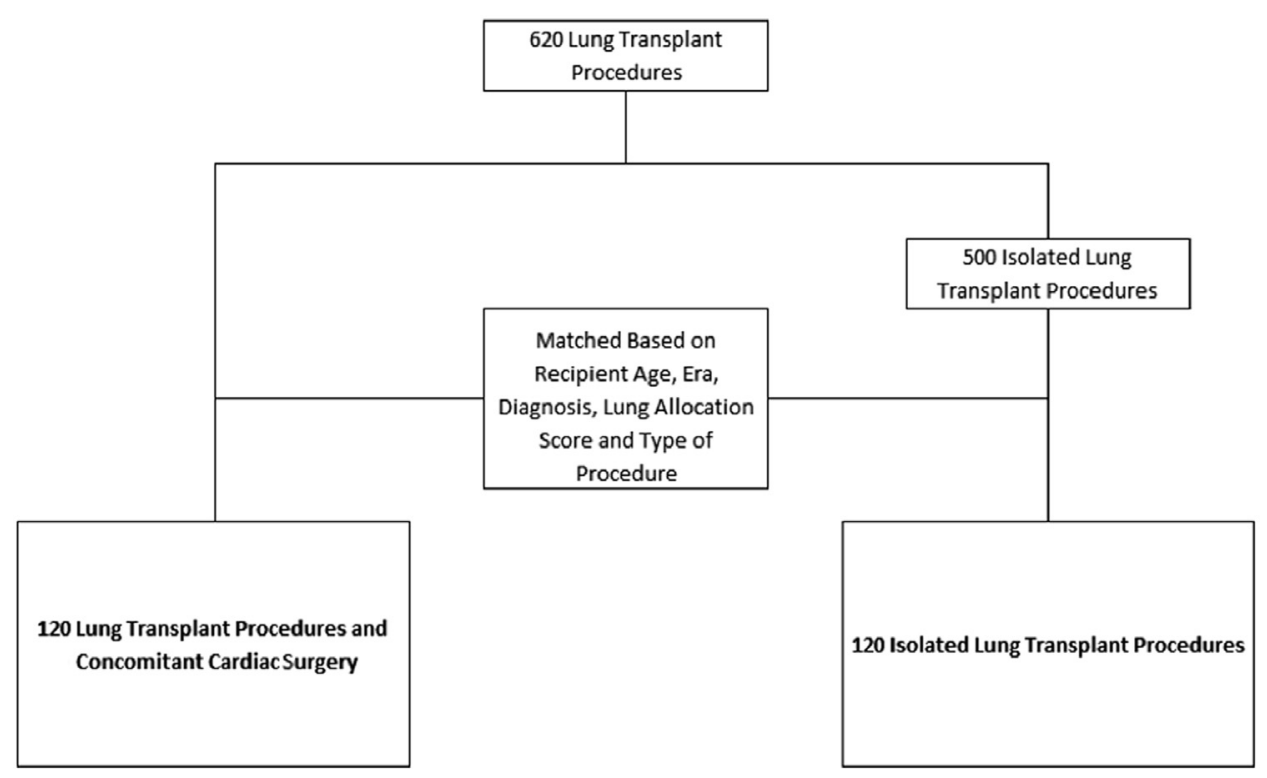

FIGURE 1. Patient flow diagram.

on the Organ Procurement and Transplantation Network 2013 annual report, which cites these variables as predictors of survival in the general lung transplant population. ${ }^{7}$ The distribution of propensity scores for our CCS population ( $\mathrm{n}=120$ ), given as median (minimum-maximum) was $0.27(0.05-0.79)$. In our control population $(n=500)$, the propensity scores were $0.14(0.00-0.65)$.

Based on these values, matching was performed, to identify the 120 controls, using STATA 6.2 software (Stata Corp, College Station, Tex). Data points are presented as mean $\pm \mathrm{SD}$, or frequency and percentage of the study population. The Kruskal-Wallis rank test was used for comparisons of continuous variables between groups; $\chi^{2}$ analysis or the Fisher exact test was used for categoric variables. Patient survival was determined using Kaplan-Meier analysis; log-rank analysis was utilized for comparisons. All statistical analyses were performed utilizing STATA 6.2 software.

\section{RESULTS}

During the study period, 120 patients underwent lung transplantation and concomitant cardiac procedures (CCS group; Figure 1). Because the total number of lung transplantation procedures performed during this period was 620 , the proportion of patients undergoing lung transplantation and CCS accounted for $19.4 \%$ of the population (Figure 2).

The donor age and body mass index were higher in the control group, compared with the CCS group (Table 1). More patients in the control group, compared with the CSS group, had a diagnosis of obstructive lung disease

Incidence of concomitant cardiac surgery

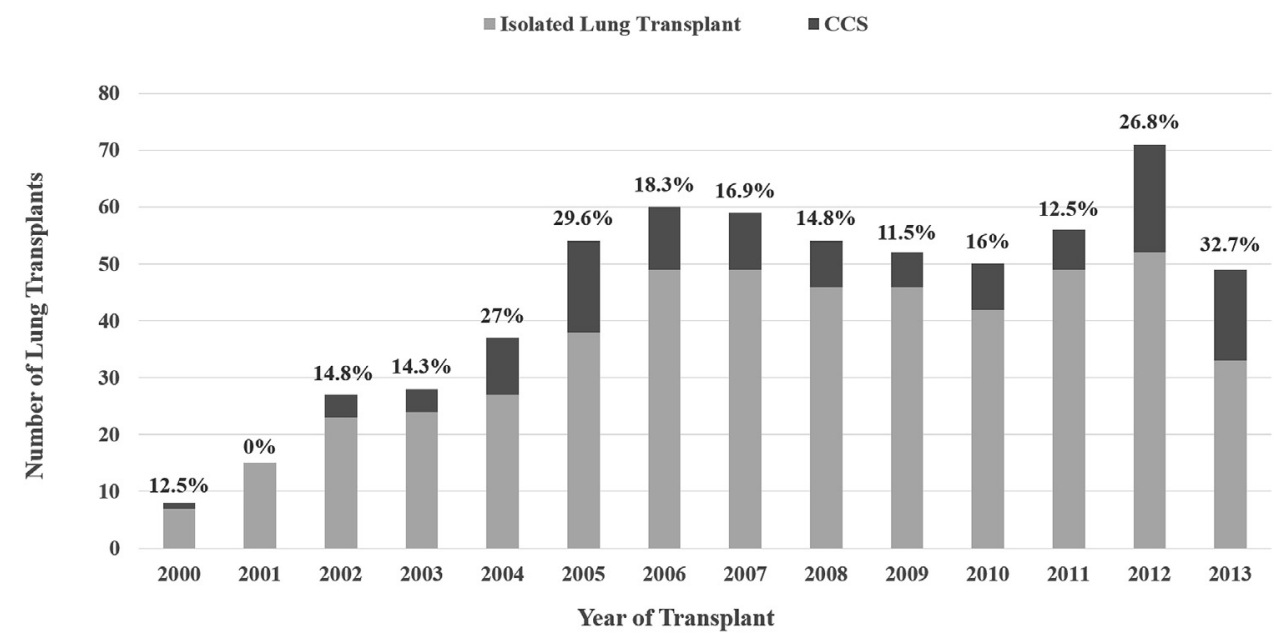

FIGURE 2. Incidence of CCS in lung transplant recipients by year of transplant. Numbers above each bar indicate the percentage of lung transplant cases that included CCS each year. CCS, Concomitant cardiac surgery. 
TABLE 1. Donor characteristics

\begin{tabular}{lccr}
\hline \multicolumn{1}{c}{ Variables } & $\begin{array}{c}\text { Control } \\
(\mathbf{n = 1 2 0})\end{array}$ & $\begin{array}{c}\text { CCS } \\
(\mathbf{n}=\mathbf{1 2 0})\end{array}$ & $\begin{array}{c}\boldsymbol{P} \\
\text { value }\end{array}$ \\
\hline Age $(\mathrm{y})$ & $38.0 \pm 15.0$ & $32.4 \pm 14.6$ & .013 \\
Gender, male & $76(59.4)$ & $81(63.3)$ & .662 \\
Body mass index $\left(\mathrm{kg} / \mathrm{m}^{2}\right)$ & $30.4 \pm 13.7$ & $26.2 \pm 11.1$ & .032 \\
Diabetes mellitus & $11(9.3)$ & $5(4.3)$ & .203 \\
Cytomegalovirus positive & $77(67.5)$ & $74(67.3)$ & .966 \\
Smoking history & $48(46.2)$ & $40(38.8)$ & .287 \\
Donor partial pressure of arterial & $443.4 \pm 105.0$ & $452.3 \pm 111.8$ & .572 \\
$\quad$ oxygen at $100 \%$ fraction of & & & \\
$\quad$ inspired oxygen & & & .055 \\
Cause of death & $17(14.3)$ & $16(13.7)$ & - \\
$\quad$ Anoxia & $55(46.2)$ & $34(29.1)$ & - \\
Cerebrovascular accident & $0(0)$ & $1(0.9)$ & - \\
Central nervous system tumor & $42(35.3)$ & $60(51.3)$ & - \\
Head trauma & $5(4.2)$ & $6(5.1)$ & - \\
Other & & & \\
\hline
\end{tabular}

Data presented as mean \pm standard deviation or number $(\%)$. Comparisons for continuous variables were made using the Kruskal-Wallis rank test and comparisons for categorical variables were made using the Pearson chi-square test. $C C S$, Concomitant cardiac surgery. *Indicates statistical significance at $P<.05$.

(Table 2). In contrast, more patients had the diagnosis of primary pulmonary hypertension in the CCS group. Additionally, patients in the CCS group had a higher pulmonary hemodynamic profile, compared with the control group. No differences were noted in lung allocation score, or days on the waitlist between groups.

No differences were found in type of transplant or incidence of cytomegalovirus and gender mismatch between donor and recipient. As expected, more patients in the CCS group had lung transplantation procedures performed on cardiopulmonary bypass. The mean cardiopulmonary bypass time and allograft ischemia time was longer in the CCS group, compared with the control group (Table 3, which additionally shows the breakdown of procedures performed in the CCS group).

The most common operation performed was a patent foramen ovale repair $(n=66)$, followed by tricuspid valve repair $(\mathrm{n}=34)$, and CABG $(\mathrm{n}=22)$. The total is $>120$, as many patients had multiple cardiac procedures. For patients who underwent coronary revascularization, the in situ left internal mammary was used for left anterior descending artery disease preferentially (when a median sternotomy was used). Venous conduits were used in the remaining patients. A total of 17 patients had 1 bypass; 4 had 2 bypasses; and the remaining 1 had 3 bypasses. Tricuspid valve repair was performed in 24 patients, using pericardial strip annuloplasty, and in 10 patients with the Edwards MC3 ring.

The median follow-up for the study population was 5.7 years, with a mean of 6.6 years. The survival of lung transplant recipients who underwent CCS was similar to that for recipients undergoing isolated lung transplantation,
TABLE 2. Recipient preoperative characteristics

\begin{tabular}{|c|c|c|c|}
\hline Variables & $\begin{array}{c}\text { Control } \\
(\mathbf{n}=\mathbf{1 2 0})\end{array}$ & $\begin{array}{c}\text { CCS } \\
(\mathbf{n}=\mathbf{1 2 0})\end{array}$ & $\begin{array}{c}P \\
\text { value }\end{array}$ \\
\hline $\operatorname{Age}(y)$ & $56.7 \pm 10.3$ & $56.6 \pm 11.3$ & .624 \\
\hline Gender, male & $79(59.8)$ & $72(54.6)$ & .350 \\
\hline Height $(\mathrm{cm})$ & $170 \pm 10.6$ & $170.7 \pm 10.5$ & .568 \\
\hline Weight $(\mathrm{kg})$ & $72.8 \pm 16.9$ & $74.6 \pm 17.0$ & .408 \\
\hline Body mass index $\left(\mathrm{kg} / \mathrm{m}^{2}\right)$ & $24.9 \pm 4.4$ & $25.3 \pm 4.3$ & .584 \\
\hline Diabetes mellitus & $22(18.3)$ & $17(14.2)$ & .382 \\
\hline Systemic hypertension & $40(3$ & $36(30)$ & .549 \\
\hline Cytomegalovirus positive & $81(67.5)$ & $76(63.3)$ & .497 \\
\hline 6-min walk test (ft) & $418.6 \pm 455.7$ & $406.5 \pm 334.2$ & .546 \\
\hline Diagnosis & & & .025 \\
\hline Obstructive lung disease & $31(25.8)$ & $24(20)$ & - \\
\hline Restrictive lung di & $80(66.7)$ & $78(65)$ & 一 \\
\hline Cystic fibrosis & (5.8) & $5(4.2)$ & - \\
\hline $\begin{array}{l}\text { Primary pulmonary } \\
\text { hypertension }\end{array}$ & $2(1.7)$ & $13(10.8)$ & - \\
\hline $\begin{array}{l}\text { Systolic pulmonary arterial } \\
\text { pressure }(\mathrm{mm} \mathrm{Hg})\end{array}$ & $42.5 \pm 16.2$ & $51.0 \pm 20.7$ & .001 \\
\hline $\begin{array}{l}\text { Diastolic pulmonary arterial } \\
\text { pressure }(\mathrm{mm} \mathrm{Hg})\end{array}$ & $18.4 \pm 8.8$ & $21.9 \pm 10.5$ & $.00 s$ \\
\hline $\begin{array}{l}\text { Mean pulmonary arterial } \\
\text { pressure }(\mathrm{mm} \mathrm{Hg})\end{array}$ & $30.5 \pm 12.5$ & $41.3 \pm 16.7$ & $<.001$ \\
\hline $\begin{array}{l}\text { Pulmonary vascular resistance } \\
\left(\text { dynes } / \mathrm{cm}^{5}\right)\end{array}$ & $310.8 \pm 244.0$ & $498.3 \pm 373.3$ & $<.001$ \\
\hline Cardiac output (L/min) & $5.2 \pm 1.2$ & $5.2 \pm 1.3$ & .975 \\
\hline Cardiac index $\left(\mathrm{L} / \mathrm{min} / \mathrm{m}^{2}\right)$ & $2.9 \pm 0.7$ & $2.8 \pm 0.7$ & .451 \\
\hline Lung allocation score & $49.2 \pm 17.4$ & $46.8 \pm 14.6$ & .610 \\
\hline Wait time (d) & $170.1 \pm 229.1$ & $157.5 \pm 188.7$ & .803 \\
\hline
\end{tabular}

Data are presented as mean $\pm \mathrm{SD}$, or $\mathrm{n}(\%)$, unless otherwise indicated. Comparisons for continuous variables were made using the Kruskal-Wallis rank test, and comparisons for categoric variables were made using the Pearson $\chi^{2}$ statistic. $C C S$, Concomitant cardiac surgery. ${ }^{*} P<.05$.

for up to 5 years (Figure 3). The survival of lung transplant recipients who had major CCS was similar to that of controls who underwent isolated lung transplantation, for up to 5 years (Figure 4).

Evaluation of perioperative clinical outcomes showed that the incidence of re-exploration for bleeding, duration of invasive mechanical ventilation, need for postoperative extracorporeal membrane oxygenation, and incidence of primary graft dysfunction III at 72 hours were similar between the 2 groups (Table 4). Moreover, the duration of intensive care unit and of hospital stay was similar between the 2 groups as well. The incidence of atrial fibrillation in the CCS and control groups was $18.9 \%$ and $21.7 \%$, respectively. None of the patients developed a myocardial infarction or cerebrovascular accident after the lung transplantation procedure. Subgroup analysis of major CCS did not show any difference in perioperative clinical outcomes, compared with the control group.

Given the underlying cardiac condition of the CCS group, an important factor to assess was whether any medium-term cardiac-related adverse events occurred that might have 
TABLE 3. Transplant characteristics

\begin{tabular}{lccc}
\hline \multicolumn{1}{c}{ Variables } & $\begin{array}{c}\text { Control } \\
(\mathbf{n = 1 2 0})\end{array}$ & $\begin{array}{c}\text { CCS } \\
(\mathbf{n}=\mathbf{1 2 0})\end{array}$ & $\begin{array}{c}\boldsymbol{P} \\
\text { value }\end{array}$ \\
\hline Type of transplant & & & .826 \\
$\quad$ Single, right & $16(13.3)$ & $15(12.5)$ & - \\
Single, left & $19(15.8)$ & $16(13.3)$ & - \\
Double & $85(70.8)$ & $89(74.2)$ & - \\
Gender mismatch & $36(30.3)$ & $36(30.8)$ & .931 \\
Cytomegalovirus mismatch & $51(44.7)$ & $50(45.5)$ & .914 \\
Number performed on & $90(75)$ & $113(94.2)$ & $<.001^{*}$ \\
cardiopulmonary bypass & & & \\
Cardiopulmonary bypass time & $148.2 \pm 102.5$ & $193.3 \pm 81.0$ & $<.001^{*}$ \\
(min) & & & \\
Allograft ischemia time (min) & $303.2 \pm 82.7$ & $326.3 \pm 81.7$ & $.030^{*}$ \\
Aortic crossclamp time (min) & - & $32.8 \pm 32.7$ & - \\
Other procedures performed & & & - \\
CABG $\times 1$ & - & $17(14.2)$ & - \\
CABG $\times 2$ & - & $4(3.3)$ & - \\
CABG $\times 3$ & - & $1(0.8)$ & - \\
Patent foramen ovale repair & - & $66(55.0)$ & - \\
Tricuspid valve repair & - & $34(28.3)$ & - \\
Mitral valve repair & - & $2(1.7)$ & - \\
Pulmonic valve repair & - & $1(0.8)$ & - \\
Aortic valve repair & - & $2(1.7)$ & - \\
Modified Maze procedure & - & $16(13.3)$ & - \\
Left atrial appendage ligation & - & $6(5)$ & - \\
Ascending aortic aneurysm & - & $2(1.7)$ & - \\
$\quad$ repair & - & $1(0.8)$ & - \\
Removal of right atrial mass & - & $29(24.2)$ & - \\
Multiple cardiac procedures & - & & \\
\hline & & & \\
\hline
\end{tabular}

Data are presented as mean $\pm \mathrm{SD}$, or $\mathrm{n}(\%)$. Comparisons for continuous variables were made using the Kruskal-Wallis rank test, and comparisons for categoric variables were made using the Pearson $\chi^{2}$ statistic. CCS, Concomitant cardiac surgery; $C A B G$, coronary artery bypass grafting. $* P<.05$.

affected survival or resource utilization. We therefore examined the 5-year incidence of major adverse cardiac events, in both groups (Table 5). Five patients in the CCS group, and 4 in the control group, were readmitted because

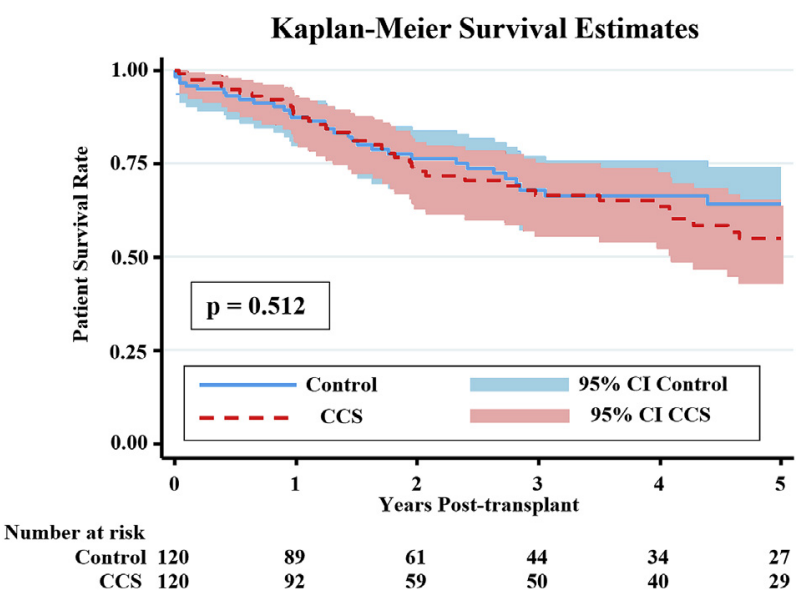

FIGURE 3. Survival curves of lung transplant recipients, with and without CCS. CCS, Concomitant cardiac surgery; $C I$, confidence interval.

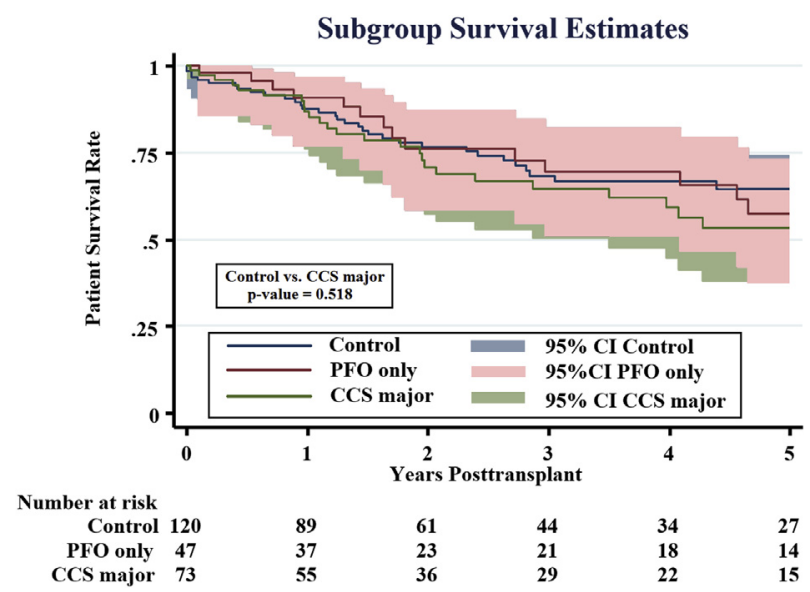

FIGURE 4. Survival curves of lung transplant recipients with major CCS versus lung transplant recipients with isolated $\mathrm{PFO}$ repair, and isolated lung transplant recipients. CCS, Concomitant cardiac surgery; $P F O$, patent foramen ovale; $C I$, confidence interval.

of complications from atrial fibrillation. One patient in the CCS group, who underwent CABG, had an acute coronary syndrome and received subsequent stent placement. One patient in the CCS group, who had undergone patent foramen ovale repair, was readmitted for congestive heart failure secondary to atrial fibrillation, which was treated medically. Overall, adverse cardiac events up to 5 years posttransplantation were rare, and no significant differences were noted between groups. Subgroup analysis of major adverse cardiac events, in the CCS group, showed no significant differences compared with the control group.

\section{DISCUSSION}

The findings of this study show that in a highly selected group of lung transplant candidates who have a surgically correctable cardiac lesion, lung transplantation and CCS can be performed with similar perioperative complication rates and medium-term survival, as compared to a cohort of isolated lung transplant recipients.

The body of data on recipient risk factors that affect the outcome of lung transplantation is increasing. . $^{7-9}$ Refinement of recipient acceptance criteria is important, because the number of donors is limited. Advanced recipient age was among the first criteria tested. Several reports have shown that in selected older patients, lung transplantation can be performed safely, with acceptable short- and medium-term outcomes. ${ }^{10,11}$

With the increasing maturity of the field, other recipient factors are being questioned. Several studies have examined the safety and feasibility of lung transplantation in patients who have coronary artery disease. ${ }^{12,13}$ Some of these patients had undergone pretransplantation percutaneous coronary intervention, and many had required concomitant CABG. Sherman and colleagues ${ }^{3}$ reported on 
TABLE 4. Perioperative clinical outcomes

\begin{tabular}{|c|c|c|c|c|c|}
\hline Variables & $\begin{array}{c}\text { Control } \\
(\mathbf{n}=120) \\
\end{array}$ & $\begin{array}{c}\text { CCS } \\
(n=120)\end{array}$ & $\begin{array}{c}\text { CCS vs control } \\
P \text { value } \\
\end{array}$ & $\begin{array}{c}\text { Major CCS } \\
(\mathbf{n}=71) \\
\end{array}$ & $\begin{array}{l}\text { Major CCS vs } \\
\text { control } P \text { value }\end{array}$ \\
\hline Duration of invasive mechanical ventilation (d) & $2(1-3)$ & $2(1-4)$ & .110 & $2(1-4)$ & .083 \\
\hline Re-exploration for bleeding & $10(8.3)$ & $17(12.9)$ & .153 & $9(12.7)$ & .333 \\
\hline Wound revision & $2(1.7)$ & $2(1.7)$ & 1.0 & $0(0)$ & .530 \\
\hline Postoperative ECMO & $3(2.5)$ & $3(2.5)$ & 1.0 & $2(2.8)$ & 1.0 \\
\hline PGD, grade III @ 72 h & $11(9.6)$ & $10(9.3)$ & .956 & $5(7.0)$ & .609 \\
\hline Intensive care unit stay (d) & $4(2-7)$ & $4(3-8)$ & .423 & $5(2-8)$ & .321 \\
\hline Hospital length of stay (d) & $15(11-24)$ & $16(12-26)$ & .276 & $16(12-23)$ & .525 \\
\hline 30-d mortality & $5(4.2)$ & $1(0.8)$ & .213 & $1(1.4)$ & .415 \\
\hline \multicolumn{6}{|l|}{ In-hospital cardiac complications } \\
\hline Atrial fibrillation & $26(21.7)$ & $25(18.9)$ & .875 & $16(22.5)$ & .889 \\
\hline Myocardial infarction & $0(0)$ & $0(0)$ & 1.0 & $0(0)$ & 1.0 \\
\hline Cerebrovascular accident & $0(0)$ & $0(0)$ & 1.0 & $0(0)$ & 1.0 \\
\hline Pericarditis & $1(0.8)$ & $0(0)$ & 1.0 & $0(0)$ & 1.0 \\
\hline
\end{tabular}

Data are presented as median (interquartile range), or $\mathrm{n}(\%)$, unless otherwise indicated. Comparisons for continuous variables were made using the Kruskal-Wallis rank test; comparisons for categoric variables were made using the Pearson $\chi^{2}$ statistic, or the Fisher exact test. All comparisons were made between the indicated subgroup and the control group. CCS, Concomitant cardiac surgery; $E C M O$, extracorporeal membrane oxygenation; $P G D$, primary graft dysfunction.

27 patients who had undergone concomitant CABG or pretransplantation percutaneous coronary intervention. Medium-term survival of this cohort was similar to that for historical cohorts. Moreover, the incidence of perioperative adverse events was similar as well.

More recently, Castleberry and colleauges ${ }^{12}$ reported on the clinical outcomes of 49 patients who underwent concomitant CABG, 38 patients who underwent pretransplantation percutaneous coronary interventions, and another 791 patients who received isolated lung transplants. ${ }^{12}$ Early mortality and adjusted hazard ratios for the cumulative risk of death were similar among all groups. However, the group who underwent lung transplantation with concomitant $\mathrm{CABG}$ had longer ventilation time, and intensive care unit and hospital stay, compared with other groups. Parekh and colleagues ${ }^{14}$ compared the clinical outcome of 35 patients who underwent lung transplantation and CCS with 665 patients who underwent isolated lung transplantation. Of the 35 patients, 27 had either patent foramen ovale or atrial septal defect repair; 4 had undergone concomitant CABG. Similar survival was observed, for up to 5 years, yet the ventilator time and intensive care unit stay were longer in the CCS group.
This study adds to the current body of literature by showing that adding a cardiac surgical procedure requiring cardiopulmonary bypass, and possible crossclamp, does not adversely affect the medium survival of a selected group of lung transplant recipients. In contrast to the findings of Castleberry and colleagues ${ }^{12}$ and Parekh and colleagues, ${ }^{14}$ we did not find that CCS with lung transplantation resulted in a higher level of perioperative use of resources. This result may be due to our stringent recipient selection criteria. When we compared the major CCS group, in isolation, to their matched control lung transplant recipients, the perioperative clinical outcomes were similar, as was the incidence of major adverse cardiac events.

In contrast to the most current body of literature, which refers to concomitant coronary revascularization in lung transplant recipients, this study includes all patients who needed CCS $(n=120)$. The findings of this study suggest that corrective cardiac procedures can be performed in lung transplant recipients, with acceptable short- and medium-term outcomes. The inference from this result and the studies cited is that we can expand the lung transplant recipient pool to include selected patients who have surgically correctable cardiac lesions.

TABLE 5. Major adverse cardiac events, for up to 5 years posttransplantation

\begin{tabular}{|c|c|c|c|c|c|}
\hline Variables & Control $(n=120)$ & $\operatorname{CCS}(n=120)$ & $\begin{array}{c}\text { CCS vs control } \\
P \text { value }\end{array}$ & Major CCS $(n=71)$ & $\begin{array}{l}\text { Major CCS vs } \\
\text { control } P \text { value }\end{array}$ \\
\hline Major adverse cardiac events & $5(4.2)$ & $6(5.0)$ & .758 & $4(5.6)$ & .729 \\
\hline Atrial fibrillation & $4(3.3)$ & $5(3.8)$ & 1.0 & $3(4.2)$ & .712 \\
\hline Acute coronary syndrome & $0(0)$ & $1(0.8)$ & 1.0 & $1(1.4)$ & .372 \\
\hline Redo revascularization & $0(0)$ & $1(0.8)$ & 1.0 & $1(1.4)$ & .372 \\
\hline Redo valve procedure & $0(0)$ & $0(0)$ & 1.0 & $0(0)$ & 1.0 \\
\hline Congestive heart failure & $0(0)$ & $1(0.8)$ & 1.0 & $0(0)$ & 1.0 \\
\hline Pulmonary embolism & $1(0.8)$ & $0(0)$ & 1.0 & $0(0)$ & 1.0 \\
\hline
\end{tabular}

Data are presented as $\mathrm{n}(\%)$, unless otherwise indicated. Comparisons for variables were made using the Pearson $\chi^{2}$ statistic, or the Fisher exact test. All comparisons were made between the indicated CCS subgroup and the control group. CCS, Concomitant cardiac surgery. 
However, continued expansion of recipient criteria in the context of a limited organ supply and hundreds of annual deaths of those on the organ waiting $\operatorname{list}^{7}$ is of questionable scientific and ethical value. We believe that expansion of the donor pool through increased public awareness, legislation, improved donor management, and adjustment of donor acceptance criteria remain major challenges facing the field of organ transplantation. Lung donation after death from cardiac-related causes may expand the donor pool in the near future. ${ }^{15}$ Ex vivo lung perfusion is a new technology that holds promise for expansion of the donor pool. ${ }^{16,17}$ Only through expansion of the donor pool can lung transplantation be offered to more patients.

This study has several limitations. The study is retrospective, and was conducted at a single institution, and therefore carries all the confounders inherent in this design. The mean follow-up time was limited to 6.6 years. The recipients were highly selected and thus, results may not be applicable to the broader population of lung transplant recipients. Quality-of-life outcome measures were not recorded. Moreover, we do not have data on patients who were evaluated for lung transplantation but deemed unacceptable as candidates.

In conclusion, lung transplant patients with cardiac diagnoses who underwent CCS and lung transplantation at our institution had similar early- and medium-term clinical outcomes, compared with isolated lung transplant recipients. The findings of this study suggest that offering lung transplantation to a highly selected group of patients who need CCS is justified. Continued expansion of the recipient pool within the context of a limited supply of donor organs warrants further study and discussion.

\section{Conflict of Interest Statement}

David Ross reports consulting fees and grant support from Actelion, as well as equity ownership in Actelion. All other authors have nothing to disclose with regard to commercial support.

You can watch a Webcast of this AATS meeting presentation by going to: http://webcast.aats.org/2015/Video/ Tuesday/04-28-15_607_0820_Biniwale.mp4.

\section{References}

1. Reed RM, Eberlein M, Girgis RE, Hashmi S, Iacono A, Jones S, et al. Coronary artery disease is under-diagnosed and under-treated in advanced lung disease. Am J Med. 2012;125:1228.

2. Snell GI, Richardson M, Griffiths AP, Williams TJ, Esmore DS. Coronary artery disease in potential lung transplant recipients $>50$ years old: the role of coronary intervention. Chest. 1999;116:874-9.

3. Sherman W, Rabkin DG, Ross D, Saggar R, Lynch JP, Ardehali A, et al. Lung transplantation and coronary artery disease. Ann Thorac Surg. 2011;92: 303-8.

4. Lee R, Meyers BF, Sundt TM, Trulock EP, Patterson GA. Concomitant coronary artery revascularization to allow successful lung transplantation in selected patients with coronary artery disease. J Thorac Cardiovasc Surg. 2002;124: 1250-1.
5. Patel VS, Palmer SM, Messier RH, Davis RD. Clinical outcome after coronary artery revascularization and lung transplantation. Ann Thorac Surg. 2003;75: 372-7; discussion 377.

6. Christie JD, Carby M, Bag R, Corris P, Hertz M, Weill D. Report of the ISHLT Working Group on Primary Lung Graft Dysfunction Part II: Definition. A Consensus Statement of the International Society for Heart and Lung Transplantation. J Heart Lung Transplant. 2005;24:1454-9.

7. Valapour M, Skeans MA, Heubner BM, Smith JM, Hertz MI, Edwards LB, et al. OPTN/SRTR 2013 Annual Data Report: lung. Am J Transplant. 2015;15(Suppl 2): $1-28$.

8. Shino MY, Lynch JP, Fishbein MC, McGraw C, Oyama J, Belperio JA, et al. Sarcoidosis-associated pulmonary hypertension and lung transplantation for sarcoidosis. Semin Respir Crit Care Med. 2014;35:362-71.

9. Ahmad U, Wang Z, Bryant AS, Kim AW, Kukreja J, Mason DP, et al. Outcomes for lung transplantation for lung cancer in the United Network for Organ Sharing Registry. Ann Thorac Surg. 2012;94:935-40; discussion 940-1.

10. Bruckner BA, Motomura T, Kaleekal TS, Jyothula SS, Scheinin S, Bunge RR, et al. Clinical outcomes in elderly lung transplant recipients 70 years and older. J Heart Lung Transplant. 2013;32:S266-7.

11. Kilic A, Merlo CA, Conte JV, Shah AS. Lung transplantation in patients 70 years old or older: have outcomes changed after implementation of the lung allocation score? J Thorac Cardiovasc Surg. 2012;144:1133-8.

12. Castleberry AW, Martin JT, Osho AA, Hartwig MG, Hashmi ZA, Zanotti G, et al. Coronary revascularization in lung transplant recipients with concomitant coronary artery disease. Am J Transplant. 2013;13:2978-88.

13. Zanotti G, Hartwig MG, Castleberry AW, Martin JT, Shaw LK, Williams JB, et al. Preoperative mild-to-moderate coronary artery disease does not affect long-term outcomes of lung transplantation. Transplantation. 2014;97: 1079-85.

14. Parekh K, Meyers BF, Patterson GA, Guthrie TJ, Trulock EP, Damiano RJ Jr, et al. Outcome of lung transplantation for patients requiring concomitant cardiac surgery. J Thorac Cardiovasc Surg. 2005;130:859-63.

15. Krutsinger D, Reed RM, Blevins A, Puri V, De Oliveira NC, Zych B, et al. Lung transplantation from donation after cardiocirculatory death: a systematic review and meta-analysis. J Heart Lung Transplant. 2015;34:675-84.

16. Machuca TN, Mercier O, Collaud S, Tikkanen J, Krueger T, Yeung JC, et al. Lung transplantation with donation after circulatory determination of death donors and the impact of ex vivo lung perfusion. Am J Transplant. 2015;15: 993-1002.

17. Mohite P, Sabashnikov A, García Sáez D, Pates B, Zeriouh M, De Robertis F, et al. Utilization of the Organ Care System Lung for the assessment of lungs from a donor after cardiac death (DCD) before bilateral transplantation. Perfusion. 2015;30:427-30.

Key Words: lung transplant, cardiac surgery, outcomes

\section{Discussion}

Dr S. Moffatt-Bruce (Columbus, Ohio). Thank you for your paper in advance. You answered many of my questions just in your presentation, but I do have one pressing question, which is a little bit more global and perhaps very important in this era of health care transformation.

Your results are superb. You did 620 transplants in 13 years, sometimes as many as 70 or more transplantations per year. Your patients are highly selected for, by virtue of their protocols and ruling in and out cardiac and thoracic disease, and I would suggest that your surgeons are also highly selected for. Therefore, do your data support the idea of regionalization? Do your data and programmatic development support the idea that we should be regionalizing and selecting for centers of excellence where highrisk, high-cost procedures should be performed, instead of dispersing them among the 73 current transplantation 
centers? So, in essence, the question is: Should all thoracic transplantation surgeons and centers be driving somewhat outside of the preconceived guardrails in this era of health care transformation?

Dr R. Biniwale (Los Angeles, Calif). Thank you very much.

Dr Moffatt-Bruce. Thank you for reviewing our paper. I think your question shows a lot of insight into the global problem of lung transplantation. At our center, in our hands, we have these results. Having said that, I do not know that they can be extrapolated to all centers. Again, the idea of a tertiary referral center, or a center of excellence, raises a lot of feelings in people.

But, what we can say definitely here is that these are our results, and I am not sure that they can be reproduced at centers that have lower volume or are not comfortable with $\mathrm{CCS}$ at the time of lung transplantation.

Dr M. Bacchetta (New York, NY). I think Marcelo has the same question about the extent of your tricuspid valve work. What was the etiology, and do you really feel the need for that? Additionally, with small patent foramen ovales, we usually do not do anything with them. So some sense of what you are correcting is needed. With an atrial septal defect, we would do a correction.

Dr Biniwale. The concern at our center has been cerebral emboli from the patent foramen ovale. We have had a few patients who did suffer an embolus, and so it has been our practice to close all patent foramen ovales, as well as atrial septal defects.

Tricuspid valve repair was performed when the annulus was $>4 \mathrm{~cm}$, or if the indexed area was $>2.5 \mathrm{~cm}^{2} / \mathrm{m}^{2}$, but usually annuloplasty was performed. Again, we have a low threshold for doing that, because the patients are already on bypass.

Dr Acker. The patients are already on bypass?

Dr Biniwale. Yes.

Dr M. Cypel (Toronto, Ontario, Canada). I just want to make a comment. It is not that you are operating on higher-risk patients, just that you are doing more cardiac repairs in patients for whom other centers would not do anything. Our incidence of doing cardiac surgery is $<2 \%$ of our transplantations, not $20 \%$, and I think most centers are in a much lower range, even though they are treating the same sort of population.

Readers who found these articles interesting may also like to read the following papers found in recent and future issues of our sister publications, Seminars in Thoracic and Cardiovascular Surgery and Operative Techniques in Thoracic and Cardiovascular Surgery!

\section{Transplantation}

Original Submission: Trends in U.S. Extracorporeal Membrane Oxygenation Use and Outcomes: 2002-2012. Fenton H McCarthy. Semin Thorac Cardiovasc Surg 2015; Summer; 27(2):81-88.

Editorial Commentary: Is it Worth it to Increase the Use of Extracorporeal Membrane Oxygenation? Marco Morsolini. Semin Thorac Cardiovasc Surg 2015; Summer; 27(2):89-90.

Original Submission: Increased Procurement of Thoracic Donor Organs After Thyroid Hormone Therapy. Dimitri Novitzky. Semin Thorac Cardiovasc Surg 2015; Summer; 27(2):123-133.

Editorial Commentary: Optimizing Donor Quality Via Hormonal Therapy: An Important Strategy to Increase Successful Placement of Thoracic Organs in End-Stage Patients. Syed M. Peer. Semin Thorac Cardiovasc Surg 2015; Summer; 27(2):133-134.

State of the Art: The State of the Art in Corporeal Membrane Oxygenation. Steve K. Singh. Semin Thorac Cardiovasc Surg 2015; Spring; 27(1):17-23.

Median Sternotomy for Lung Transplantation. John Dark. Oper Tech Thorac Cardiovasc Surg 2015; Spring; 20(1):87-103.

Less Invasive Techniques for Left Ventricular Assist Device Implantation. Scott C. Silvestry. Expected Publication December 2015.

Ex Vivo Lung Perfusion. Marcelo Cypel. Oper Tech Thorac Cardiovasc Surg 2014; Winter, 19(4): 433-442.

Subcostal Approach to Replacement of a HeartMate II Device: Indications and Technique. Nader Moazami. Oper Tech Thorac

Cardiovasc Surg 2014; Winter, 19(4):443-453. 